\title{
Restoration of Esthetics and Function in a Patient with Amelogenesis Imperfecta
}

\author{
1Jasmeet Singh Chandhok, ${ }^{2}$ Deepika Thosre Chandhok, ${ }^{3}$ Vaibhao Ishwar Shambharkar
}

\begin{abstract}
An unpleasant smile will not only hamper the looks of the person but also leaves a social withdrawal from the society specially for teenagers. Amelogenesis imperfecta $(\mathrm{Al})$ is one such condition which affects not only the teeth but to overall social and psychological growth of a person. This clinical case deals with step-by-step procedures involved with multidisciplinary approach to deal with such cases in a short period of time with reduced financial burden over the patient.

Amelogenesis imperfecta is a heterogeneous inherited disorder that disturbs the developing enamel structure and exists independent of any related systemic disorder. It affects both the primary and permanent dentitions. Transmission of the gene is autosomal dominant, recessive or X-linked mode of inheritance.

Clinical findings of Al include: Enamel deficiencies, pulpal calcification, root malformations, failed tooth eruption, impaction of permanent teeth, progressive root and crown resorption, congenitally missing teeth, tooth sensitivity. Due to reduced enamel thickness, the teeth are undersized, with lack of contact between adjacent teeth, poor oral hygiene and mouth breathing with associated gingivitis and gingival hyperplasia may also be seen.
\end{abstract}

Keywords: Amelogenesis imperfecta, Esthetics, Full mouth rehabilitation.

How to cite this article: Chandhok JS, Chandhok DT, Shambharkar VI. Restoration of Esthetics and Function in a Patient with Amelogenesis Imperfecta. Int J Prosthodont Restor Dent 2015;5(4):118-121.

Source of support: Nil

Conflict of interest: None

\section{INTRODUCTION}

Amelogenesis imperfecta (AI) is a condition which affects not only the teeth but to overall social and psychological growth of a person. The incidence of AI has been reported to vary between approximately 1:700

\footnotetext{
${ }^{1,2}$ Associate Professor, ${ }^{3}$ Assistant Professor

1,3 Department of Prosthodontics, Swargiya Dadasaheb Kalmegh Smruti Dental College and Hospital, Nagpur, Maharashtra, India

${ }^{2}$ Department of Endodontics and Conservative Dentistry Swargiya Dadasaheb Kalmegh Smruti Dental College and Hospital, Nagpur, Maharashtra, India
}

Corresponding Author: Jasmeet Singh Chandhok, Associate Professor, Department of Prosthodontics, Swargiya Dadasaheb Kalmegh Smruti Dental College and Hospital, Nagpur Maharashtra, India, Phone: 7757033775, e-mail: dentalsoln@ gmail.com and $1: 16,000$, depending on the population studied and the diagnostic criteria used. ${ }^{1-4}$ Although has been categorized into four broad groups primarily based on phenotype-hypoplastic, hypocalcified, hypomaturation, and hypomaturation-hypoplastic-at least 15 subtypes of exist when phenotype and mode of inheritance are considered..$^{5-8}$

Each case of AI is unique, and warrants careful consultation with a variety of experts to obtain the best outcome. Children with AI can exhibit accelerated tooth eruption compared to the normal population or have late eruption. Other clinical implications of AI include: low caries susceptibility, rapid attrition, excessive calculus deposition, and gingival hyperplasia. Pathologies associated with AI are enlarged follicles, impacted permanent teeth, ectopic eruption, congenitally missing teeth, crown and/or root resorption, and pulp calcification. Agenesis of second molars also has been observed. Although uncommon in AI, enamel resorption and ankylosis have been reported. In addition, the incidence of anterior open bite is $50 \%$ in hypoplastic AI, $31 \%$ in hypomaturation $\mathrm{AI}$, and $60 \%$ of hypocalcified AI.

Treating a patient with $\mathrm{AI}$ is important not only from a functional standpoint but also from a psychological point of view, and hence the aim of the treatment should be to both restore esthetics and improve masticatory function.

\section{CASE HISTORY}

A 19-year-old male previously diagnosed with hypocalcification AI presented for prosthodontic treatment. His primary concerns included extreme tooth sensitivity, dissatisfaction with the size, shape, and shade of his teeth, and poor masticatory efficiency (Fig. 1). The family medical history revealed that the patient's

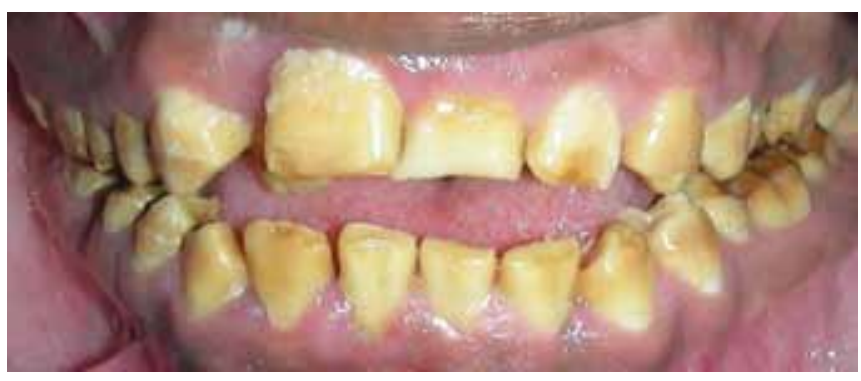

Fig. 1: Pretreatment view 
mother was affected by AI and got all her teeth extracted and replaced with complete denture. His oral hygiene was poor, tooth hypersensitivity and the presence of an anterior open bite associated with mouth breathing were also present. There may have been little or no oral healthcare during childhood and pocket depths recorded were $3 \mathrm{~mm}$ or more. The patient stated that his primary teeth were small and discolored. The severe occlusal wear with loss of vertical dimension was compensated by dentoalveolar growth. Panoramic radiographs revealed loss of enamel on occlusal surface of all the posterior teeth. The patient had retained maxillary deciduous canines on the right and left sides (Fig. 2). Provisional diagnosis was made as hypocalcified type of AI with poor esthetics.

Treatment planning includes the surgical phase where extraction of maxillary right central incisor was done followed by periodontal treatment of oral prophylaxis with crown lengthening of all teeth which is followed by endodontic phase of root canal treatment of all teeth (Fig. 3). Finally, the prosthetic phase includes restoration of all teeth with anterior teeth with metal ceramic crowns and posteriors with all metal crowns with Pankey-MannSchuyler's philosophy for full mouth rehabilitation.

Complete maxillary and mandibular arch impressions were made using irreversible hydrocolloid as diagnostic

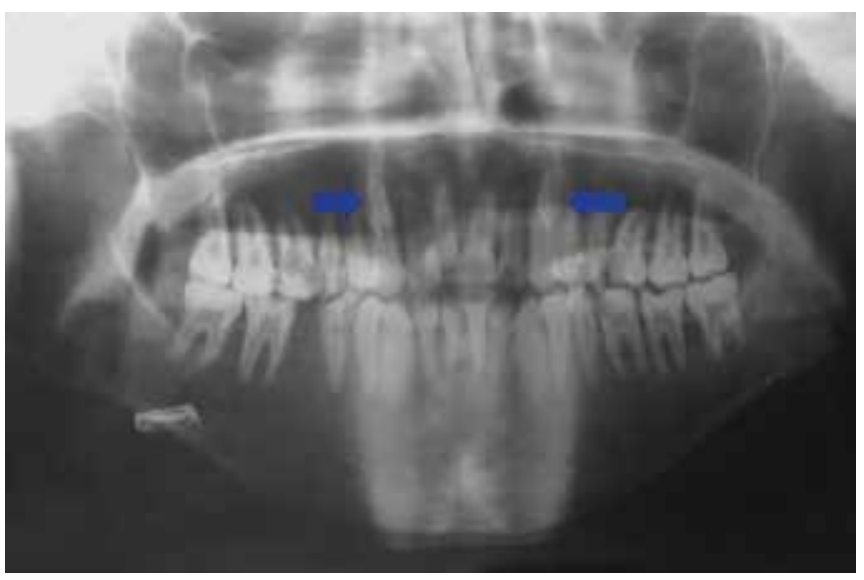

Fig. 2: Preoperative panoramic view

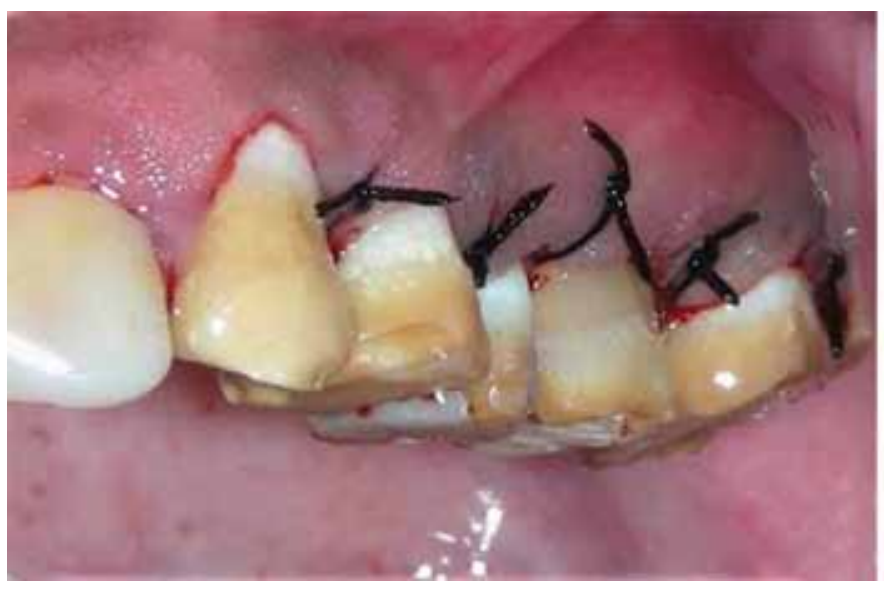

impressions. Diagnostic casts were fabricated from type III gypsum product they were mounted on a semiadjustable articulator with a face bow transfer and a centric relation record using polyvinylsiloxane occlusal registration material the articulator was programmed using protrusive and lateral records. The diagnostic preparations and wax-up revealed insufficient interocclusal space for fixed prostheses. Vertical dimension of occlusion (VDO) was increased by $4 \mathrm{~mm}$ using occlusal splint device for a period of 3 months. Niswonger's technique and phonetics were used to establish the new VDO. Diagnostic preparations and waxing on the casts were done to evaluate the amount of tooth reduction for planned metal ceramic and all metal restorations after confirming that the patient could tolerate the increase in the vertical dimension. Crown lengthening procedure was performed on both maxillary and mandibular teeth (Fig. 4). A mutually protected occlusal scheme was developed by doing a diagnostic wax-up (Fig. 5). A tooth preparation with a chamfer margin configuration for metalceramic crowns was performed for anterior teeth followed by an all chamfer margin for posterior teeth in sequential manner (Figs 6 and 7). Provisional restorations were fabricated from the diagnostic wax-up putty index and they were cemented

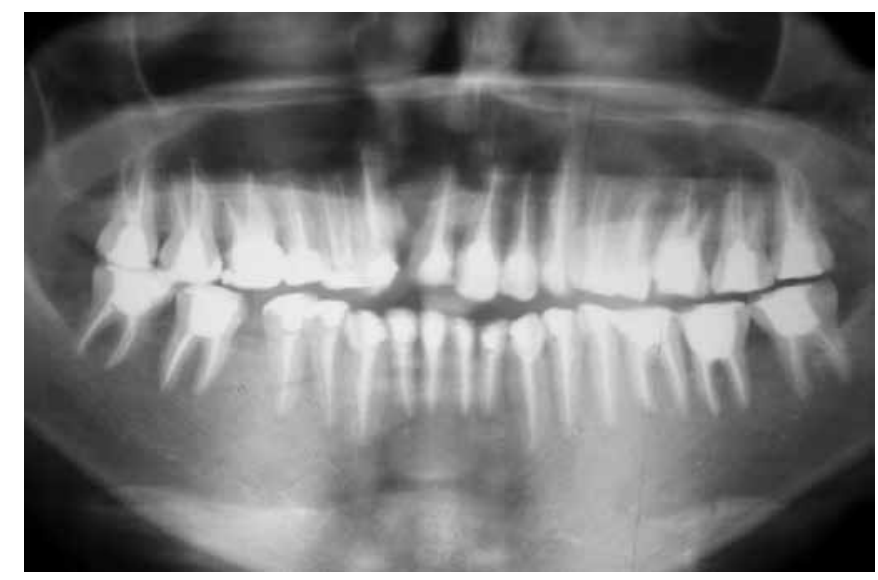

Fig. 3: Post-endodontic view

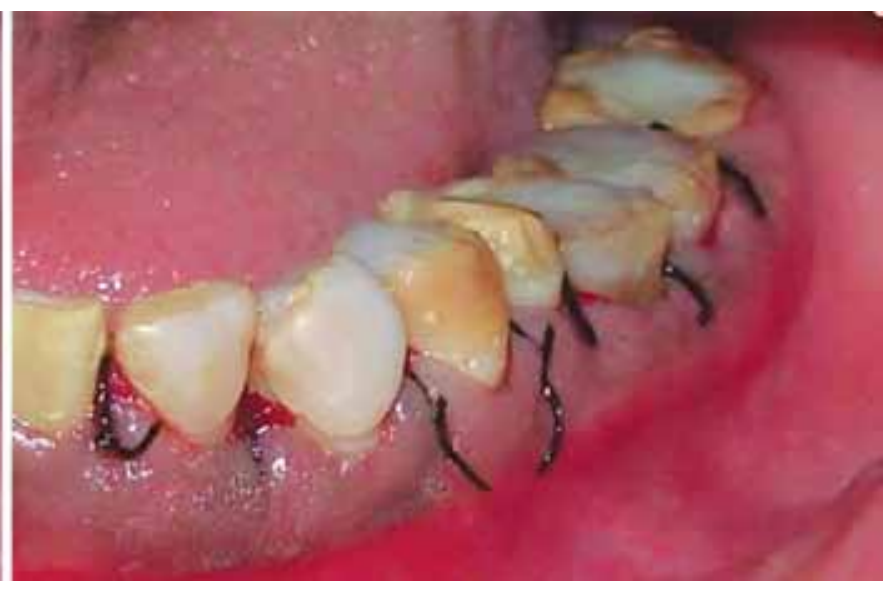

Fig. 4: Crown lengthening procedure 


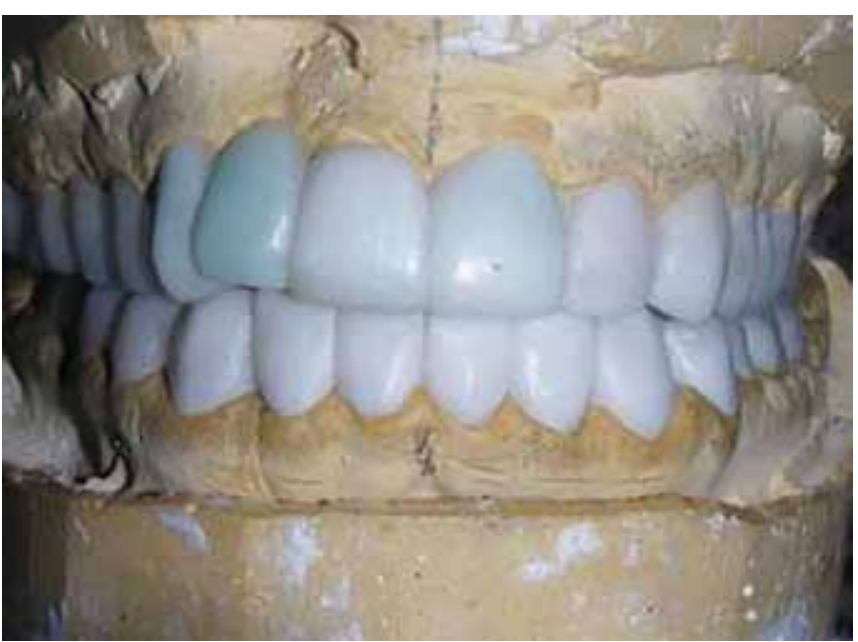

Fig. 5: Diagnostic wax-up

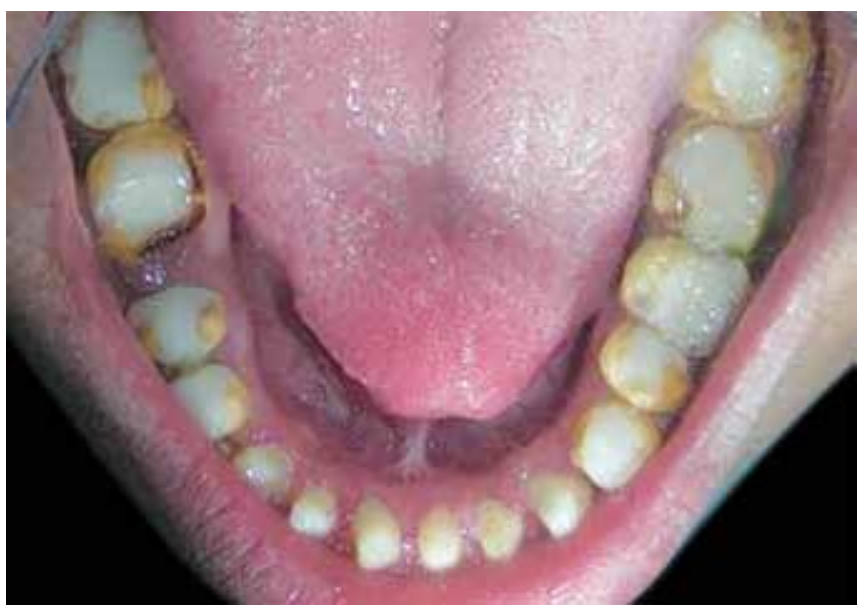

Fig. 7: Mandibular arch crown preparation

with zinc oxide eugenol temporary cement (Fig. 8). The patient wore the provisional restorations at the new vertical dimension for 4 months. A custom incisal guide table was fabricated from the acrylic resin to preserve the anterior guidance of provisional restorations for the fabrications of definitive restorations. Gingival retraction was maintained to accurately record finish lines in the definitive impressions using a knitted cord soaked in aluminum chloride. Shades for the definitive restorations were determined prior to tooth preparation and they were reviewed after tooth preparation. Definitive impressions of the prepared maxillary and mandibular anterior teeth were made using polyvinyl siloxane impression material by putty wash technique. Working casts were fabricated from type IV gypsum product and they were mounted on the semiadjustable articulator using interocclusal records. Anterior metal ceramic crowns were fabricated. The crowns were evaluated and adjusted for optimal contacts, contours, and esthetics intraorally and they were luted. On posterior teeth, all metal crowns with metal occlusal surfaces were fabricated, evaluated intraorally,

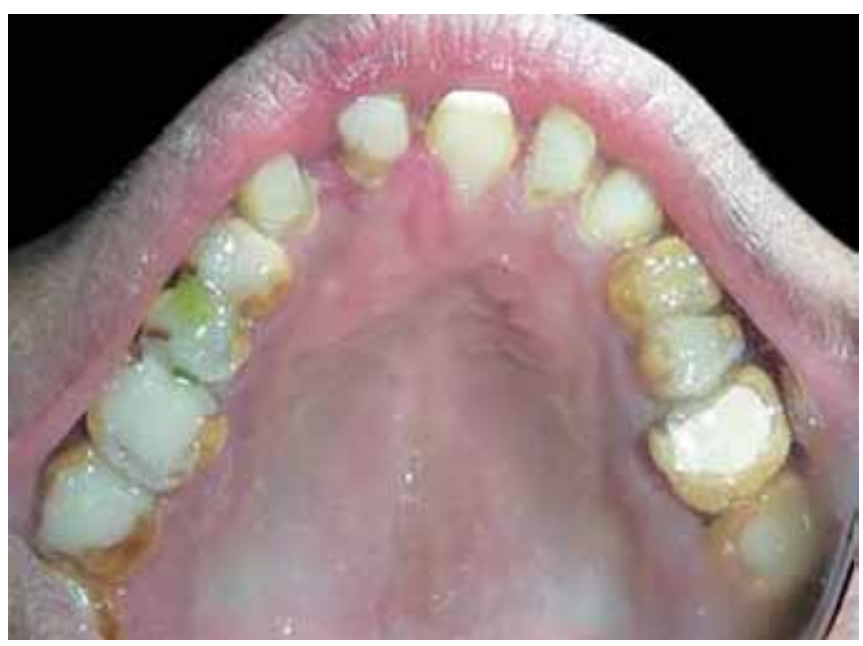

Fig. 6: Maxillary arch crown preparation

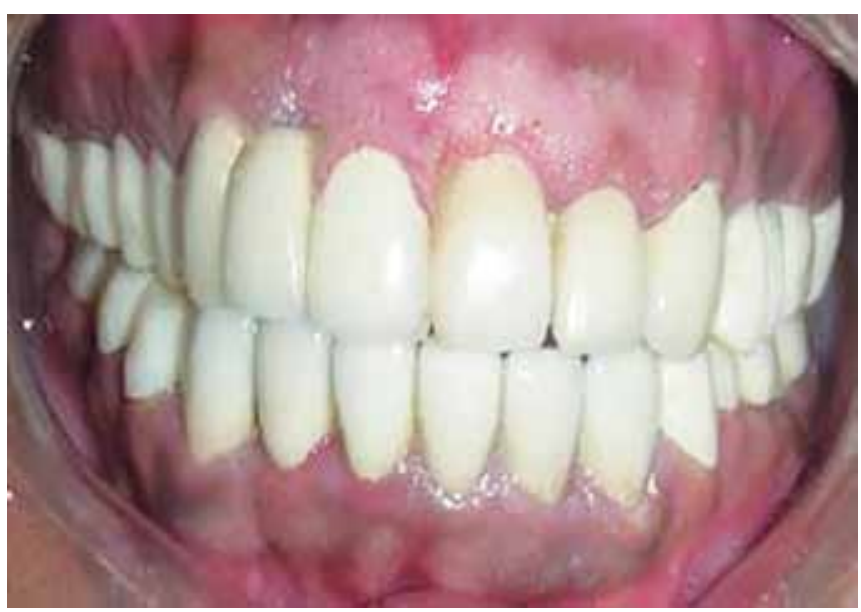

Fig. 8: Provisional restoration

adjusted, and cemented (Figs 9 and 10). The outcome of the treatment in terms of function and esthetics satisfied the expectations of patient. The patient was monitored at 6 months intervals for 1 year, and then once a year for recall, and he has not experienced any complications since past 5 years.

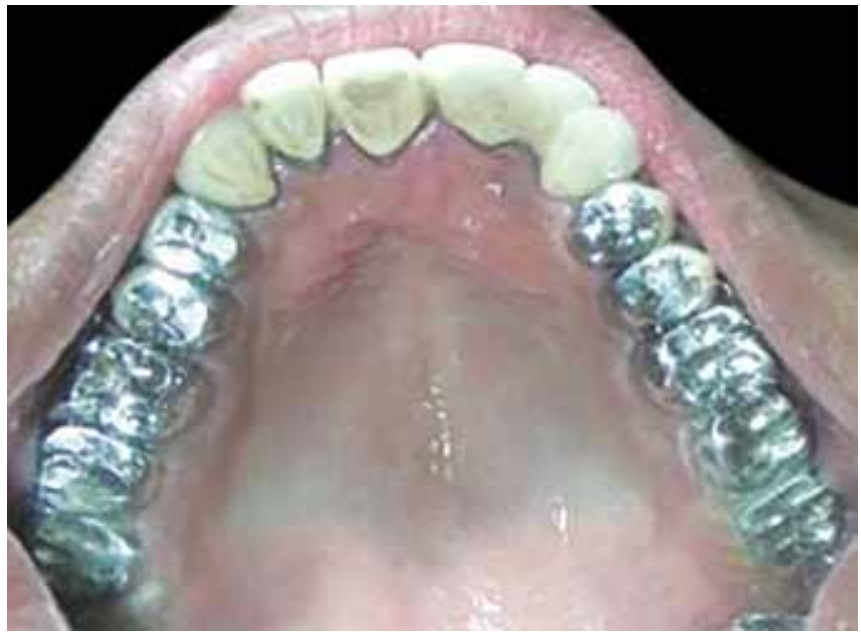

Fig. 9: Maxillary final prosthesis 


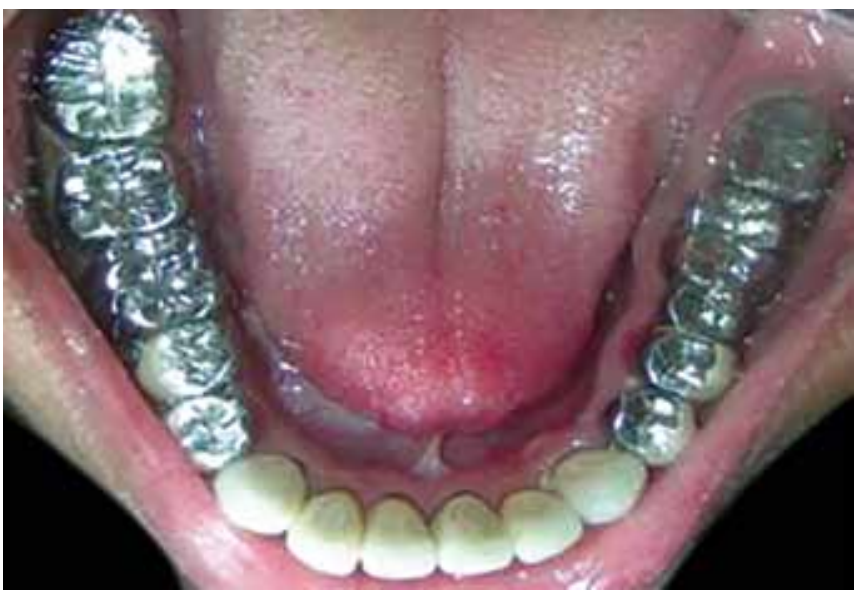

Fig. 10: Mandibular final prosthesis

\section{DISCUSSION}

When patient requires a comprehensive approach, a mutual understanding and communication among the prosthodontic, endodontic, and periodontic disciplines is very critical, to achieve the improved functional and esthetic outcome. In this complex rehabilitation, it is essential for the prosthodontist to play a key role in the multidisciplinary team, in supporting both patient and parents. Patients with AI are often esthetically and functionally affected because of tooth discoloration, with accompanying hypersensitivity and loss of VDO. Restoration of such inherited defects is essential, not only due to functional and esthetic reasons, but also because there is a positive psychological impact on young patients. Treatment planning for patients with AI is related to many factors, including age and socioeconomic status of the patient, the type and severity of the disorder and the intraoral situation at the time that the treatment is planned. Historically, treatment of patients with AI has included multiple extractions and fabrication of complete dentures. This treatment option is psychologically harsh, especially when addressing adolescent patients. ${ }^{9,10}$ For rehabilitation of patients with AI, several treatment alternatives, with different materials and methods for restorative procedures, are available. Besides, the advances in the field of esthetic dentistry, especially in bonding to dentin, help practitioners to restore function and esthetics to an acceptable level. The most predictable and durable esthetic option is to restore the affected teeth with full coverage crowns. It has been suggested that all-ceramic restorations should be bonded to tooth structure with adhesive resin cements, to enhance fracture resistance of the restorations. ${ }^{11,12}$ The clinician must carefully balance the esthetic needs of the patient, strength of the restoration, protection of remaining teeth, and long-term prognosis of the treatment. Marginal fit and color acceptability of the restorations were satisfactory in our case, which

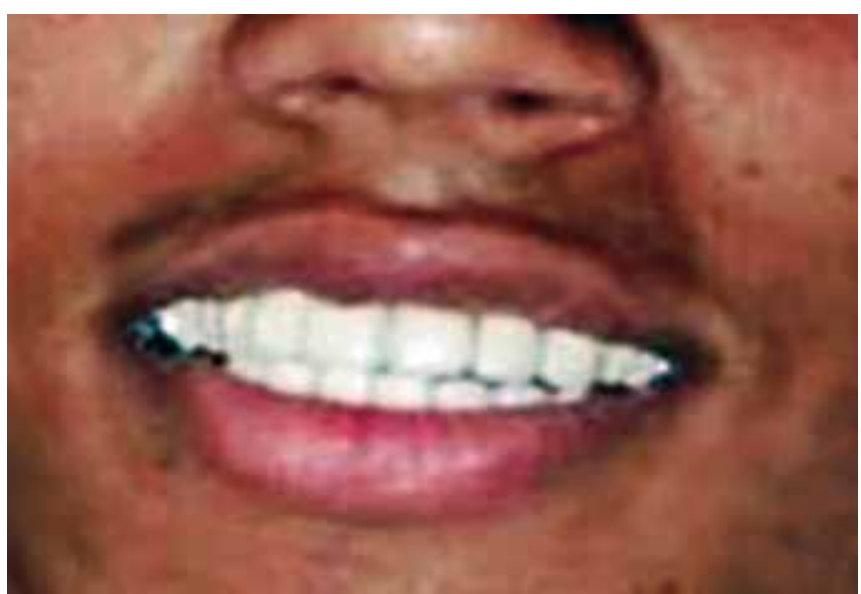

Fig. 11: A confident smile

improved the patient's self-confidence (Fig. 11). Appropriate restorative treatment is mandatory to prevent the development of psychological problems arising from the appearance of teeth affected by $\mathrm{AI}$ and bring back the patient to main stream of the social circle where they are confident to smile.

\section{REFERENCES}

1. Witkop CJ, Rao SR. Inherited defects in tooth structure. Birth Defects Orig Artic Ser 1971 Jun;7(7):153-184.

2. Backman B, Holm AK. Amelogenesis imperfecta: prevalence and incidence in a northern Swedish county. Community Dent Oral Epidemiol 1986 Feb;14(1):43-47.

3. Sundell S, Koch G. Hereditary amelogenesis imperfecta: epidemiology and clinical classification in a Swedish child population. Swed Dent J 1985;9(4):157-169.

4. Witkop CJ. Hereditary defects in enamel and dentin. Acta Genet Stat Med 1957;7(1):236-239.

5. Weinmann JP, Svoboda JF, Woods RW. Hereditary disturbances of enamel formation and calcification. J Am Dent Assoc 1945;32:397-418.

6. Sundell S, Valentin J. Hereditary aspects and classification of hereditary amelogenesis imperfecta. Community Dent Oral Epidemiol 1986 Aug;14(4):211-216.

7. Witkop CJ. Amelogenesis imperfecta, dentinogenesis imperfecta and dentin dysplasia revisited: problems in classification. J Oral Path 1988 Nov;17(9-10):547-553.

8. Aldred MJ,Crawford PJM. Variable expression in amelogenesis imperfecta with taurodontism. J Oral Pathol Med 1988 Aug;17(7):327-333.

9. Williams WP, Becker LH. Amelogenesis imperfecta: functional and esthetic restoration of severly compromised dentition. Quintessence Int 2000 Jun;31(6):397-403.

10. Lindunger A, Smedberg J. A retrospective study of the prosthodontic management of patients with amelogenesis imperfecta. Int J Prosthodont 2005 May-Jun;18(3):189-194.

11. Blatz MB. Long-term clinical success of all-ceramic posterior restorations. Quintessence Int 2002 Jun;33(6):415-426.

12. Malament KA, Socransky SS. Survival of Dicor glass-ceramic dental restorations over 16 years-part III: effect of luting agent and tooth to tooth-substitute core structure. J Prosthet Dent 2001 Nov;86(5):511-519. 